\title{
A Lack of Anticipatory Remapping of Retinotopic Receptive Fields in the Middle Temporal Area
}

\author{
Wei Song Ong ${ }^{1,3}$ and James W. Bisley ${ }^{1,2,3,4}$ \\ ${ }^{1}$ Department of Neurobiology, and ${ }^{2}$ Jules Stein Eye Institute, David Geffen School of Medicine at University of California Los Angeles, ${ }^{3}$ Interdepartmental \\ PhD Program for Neuroscience, and ${ }^{4}$ Department of Psychology and the Brain Research Institute, University of California Los Angeles, Los Angeles, \\ California 90095
}

The middle temporal (MT) area has traditionally been thought to be a retinotopic area. However, recent functional magnetic resonance imaging and psychophysical evidence have suggested that human MT may have some spatiotopic processing. To gain an understanding of the neural mechanisms underlying this process, we recorded neurons from area MT in awake behaving animals performing a simple saccade task in which a spatially stable moving dot stimulus was presented for $500 \mathrm{~ms}$ in one of two locations: the presaccadic receptive field or the postsaccadic receptive field. MT neurons responded as if their receptive fields were purely retinotopic. When the stimulus was placed in the presaccadic receptive field, the response was elevated until the saccade took the stimulus out of the receptive field. When the stimulus was placed in the postsaccadic receptive field, the neuron only began its response after the end of the saccade. No evidence of presaccadic or anticipatory remapping was found. We conclude that gain fields are most likely to be responsible for the spatiotopic signal seen in area MT.

\section{Introduction}

When staring at a point in space, the region of a scene represented in detail is small. We make multiple fast movements with our eyes to various points in space to obtain a more comprehensive picture of the world. As this occurs, the image incident on our retina is constantly changing, but we perceive the world as stationary (Wurtz, 2008). One way that this stability is thought to occur is via the passing of information from neuron to neuron within a cortical area before or during the time of a saccade (Duhamel et al., 1992). This "remapping" removes the smear of visual information racing across the retina and provides a more stable map of the visual field. A second possibility is that a signal from the eyes (Wang et al., 2007) or from areas driving the eyes (Sommer and Wurtz, 2002) giving information about their position can be combined with the visual information to calculate the locations of objects in a spatial reference frame (Zipser and Andersen, 1988). Such a signal is thought to be present in a number of visual areas in the form of gain fields (Andersen and Mountcastle, 1983; Bremmer et al., 1997; Bremmer, 2000). A third and more idealized way to accomplish this stability would be if some neurons were spatiotopic, i.e., their frames of reference are tied to a fixed reference point in the world, and the information they encode

Received 0ct. 20, 2010; revised May 12, 2011; accepted May 17, 2011.

Author contributions: W.S.O. and J.B. designed research; W.S.0. performed research; W.S.0. and J.B. analyzed data; W.S.O. and J.B. wrote the paper.

This work was supported by a Klingenstein Fellowship Award in the Neurosciences, an Alfred P. Sloan Foundation Research Fellowship, a McKnight Scholar Award and the National Eye Institute (R01 EY019273-01). We thank Bart Krekelberg, Till Hartmann, and Mickey Goldberg for their comments on an earlier version of this manuscript and the members of the University of California Los Angeles Division of Laboratory Animal Medicine for their superb animal care.

Correspondence should be addressed to Weisong 0ng, Department of Neurobiology, P0 Box 951763, Los Angeles, CA 90095-1763. E-mail:weisong@ucla.edu.

DOI:10.1523/JNEUROSCI.5589-10.2011

Copyright $\odot 2011$ the authors $\quad 0270-6474 / 11 / 3110432-05 \$ 15.00 / 0$ does not change when eye movements are made (Galletti et al., 1993). However, neurons in most visual areas are thought to be strictly retinotopic, i.e., their frames of reference are tied to a fixed point on the retina, so every eye movement brings new stimuli into the receptive field.

The middle temporal (MT) area is thought to be very important in the perception of motion, and it was generally accepted that it performed its tasks in retinotopic coordinates (Albright and Desimone, 1987; Krekelberg et al., 2003; Gardner et al., 2008). However, a recent functional magnetic resonance imaging (fMRI) study suggested that human MT has a spatiotopic processing component (d'Avossa et al., 2007), and psychophysical evidence from adaptation, perceptual learning, and integration studies appear to support this (Melcher and Morrone, 2003; Melcher, 2005; Zhang and Li, 2010) (but see Afraz and Cavanagh, 2009; Knapen et al., 2009; Morris et al., 2010). Recently, we conducted a psychophysical study on humans which used a shortterm memory for motion task that appeared to corroborate the possibility of spatiotopic processing in area MT (Ong et al., 2009). While MT neurons are known to have gain fields (Bremmer et al., 1997), it is not clear whether such activity explains the apparent spatiotopic blood oxygenation level-dependent (BOLD) signal in human MT (d'Avossa et al., 2007). In this study, we asked if the activity of MT neurons can explain the spatiotopic processing seen in these studies via presaccadic remapping or by the presence of spatiotopic receptive fields. We found that MT neurons processed information in a retinotopic coordinate frame and did not appear to exhibit preparatory remapping with a spatially and temporally stable stimulus.

\section{Materials and Methods}

Subjects. All experiments were approved by the Chancellor's Animal Research Committee at the University of California, Los Angeles as com- 

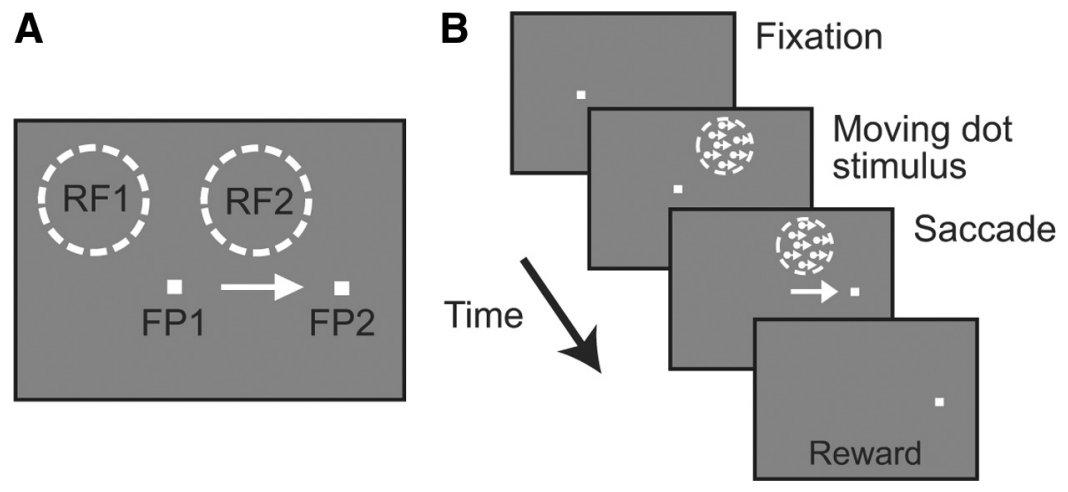

Figure 1. Task. $A$, In a given session, the positions of the initial fixation point (FP1), the saccade target (FP2), the presaccadic receptive field (RF1), and the postsaccadic receptive field (RF2) were set so that the classical retinotopic receptive field at FP1 was RF1 and the receptive field at FP2 was RF2. $\boldsymbol{B}$, In the stable moving dot stimulus task, a 500 ms coherently moving dot stimulus appeared in the receptive field $50 \mathrm{~ms}$ before FP1 was extinguished. This was typically $\sim 308 \mathrm{~ms}$ before saccadic onset.

plying with the guidelines established in the Public Health Service Guide for the Care and Use of Laboratory Animals. Two male rhesus monkeys $(8-10 \mathrm{~kg})$ were implanted with head posts, scleral search coils, and recording cylinders during sterile surgery under general anesthesia; animals were initially anesthetized with ketamine and dexdomitor and maintained with isoflurane. For details, see Mirpour et al. (2009).

Physiological recordings. We recorded extracellular single-unit activity from area MT using tungsten microelectrodes guided by coordinates from MRI images. Recorded neurons were considered to be in MT if they were found on the floor of the superior temporal sulcus, showed robust directionally selective responses to moving dot stimuli, and had receptive field sizes consistent with those found in previous studies (Desimone and Ungerleider, 1986; Albright and Desimone, 1987). After isolating the response of a single neuron, the size and position of the receptive field was mapped. The preferred direction was calculated by recording the response of the neuron to eight standard directions of motion $(0,45,90$, $135,180,225,270$, and $315^{\circ}$ from vertical) using a random dot stimulus (100\% coherence) and fitting the data with a Gaussian. The preferred direction was taken as the peak of the Gaussian fit. We discriminated action potentials during the recording epoch using the MEX pattern spike sorter (monkey E) or SortClient using the Plexon system (monkey D). The experiments were run using the REX system (Hays et al., 1982), and visual stimuli were presented on a cathode ray tube (CRT) using the associated VEX software. Eye positions were monitored using the DNI coil system and signals were collected at $1 \mathrm{kHz}$.

Behavioral task. Stimuli were presented on a Samsung SyncMaster 1100DF CRT running at $100 \mathrm{~Hz}$ situated in a dark room. The temporal precision of stimulus onset and offset was captured by a photoprobe on the corner of the monitor. To begin a trial (Fig. 1), the monkeys had to fixate a spot on the screen (FP1); they were required to maintain fixation as long as the spot was on. In most trials, the initial fixation point was extinguished after $300-800 \mathrm{~ms}$, and a new fixation point (FP2), to which the animals had to make a saccade, appeared simultaneously. The position of FP2 was chosen so that it was not in the receptive field when the animal was fixating FP1 and that its onset never produced a significant response. Once set, the positions of these two points were kept constant throughout the session (Fig. $1 \mathrm{~A}$ ). In all cases, the animals had to maintain fixation within a 3.5 deg square window. In most of the trials, a task irrelevant moving dot stimulus (100\% coherence) was presented at the presaccadic (RF1) or postsaccadic (RF2) receptive field location for 500 $\mathrm{ms}$. The stimulus was composed of $5.5 \mathrm{dots}$ per $\mathrm{deg}^{2}$ moving at $8 \mathrm{deg} / \mathrm{s} \mathrm{in}$ the preferred direction. The stimulus appeared $50 \mathrm{~ms}$ before the initial fixation point was extinguished (Fig. $1 B$ ). Given a mean saccadic latency of $258.7 \pm 56.7$, this resulted in the stimulus being presented on average for $308 \mathrm{~ms}$ before the saccade. In the remaining trials, the animal made a saccade, but no moving dot stimuli were presented.

Neural data analysis. Data were recorded from 73 MT neurons. We analyzed neural activity from all correctly performed trials. Data were aligned by stimulus onset, the beginning of the saccade, and the end of the saccade. Spike density functions were calculated by convolving spike trains with a Gaussian kernel with a $10 \mathrm{~ms} \sigma$. Data were normalized by the value of the half-height of the visual response.

To compute the latency of the neural response, we used the Poisson method described previously (Maunsell and Gibson, 1992; Bisley et al., 2004). Briefly, a Poisson distribution of the background activity was taken from the 100 $\mathrm{ms}$ before stimulus onset. The latency was taken as the first bin of three consecutive bins after the stimulus onset to have a response that was significantly greater $(p<0.01)$ than that found in the background distribution. For stimulus onset, bin sizes were $2 \mathrm{~ms}$, but for the postsaccadic responses, which were sometimes less consistent, bin sizes of 4-6 ms were occasionally used. The response-off time was defined as the time at which the spike density function first dropped below a value that was halfway between the presaccadic response and the response well after the saccade.

\section{Results}

\section{Absence of spatiotopic receptive fields in area MT}

To test whether neurons in area MT have spatiotopic or retinotopic receptive fields, we trained 2 monkeys to perform a saccade task during which a $100 \%$ coherent moving dot stimulus was presented for $500 \mathrm{~ms}$ in one of two locations. Within a session, the two fixation points (and hence the direction of the saccade) were kept constant, as were the locations of the stimuli (RF1 and RF2) (Fig. 1A). These trials were randomly interleaved with trials in which a flash could occur briefly around the time of the saccade; the results of which are described below. Before running this task, the direction tuning of the isolated neuron was recorded so that the dots in the saccade task moved in the neuron's preferred direction. We recorded the activity of 73 single MT neurons (30 from monkey D; 43 from monkey E) in this task.

All 73 neurons had retinotopic receptive fields. Figure $2 \mathrm{~A}$ (black trace) shows the recorded response of a single neuron when the stimulus was presented in RF1, while the monkey was fixating FP1. The gray bar on the abscissa represents the interval in which the stimulus was present. When a saccade from FP1 to FP2 occurred, and the stimulus was placed in RF1, the neuronal response fell off (green trace), as would be expected of a neuron with a retinotopic receptive field. Likewise, when the stimulus was presented in RF2, the main increase in visual activity occurred after the saccade had been made (blue trace), also consistent with a retinotopic receptive field. In this neuron, there was a small initial response that occurred at a slightly longer latency when the stimulus was placed in RF2. This occurred in $\sim 10 \%$ of the neurons and is consistent with the activity of MT neurons to remote stimuli (Zaksas and Pasternak, 2005).

Similar retinotopic responses were seen in all of the neurons we examined; no single neuron showed evidence of a true spatiotopic receptive field. Thus, the mean normalized population response shows a clear transition in response from RF1 to RF2 across the saccade (Fig. $2 \mathrm{~B}$ ). There was also a noticeable increase in activity as the stimulus in RF2 was brought into the receptive field (blue trace), compared with the response when the monkey was fixating FP1 and the stimulus was present in RF1 (black trace). This is probably because the response of the neuron to the stimulus in RF1 had adapted after $\sim 300 \mathrm{~ms}$ of presentation, so the initial response to a stimulus brought into the receptive field 

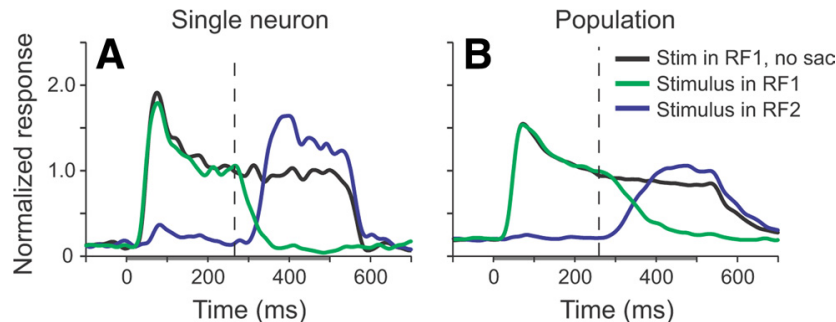

Figure 2. Mean normalized responses of MT neurons to the stable moving dot stimulus. $A$, An example MT neuron. $\boldsymbol{B}$, The mean normalized response from 73 MT neurons from two monkeys. The gray bar under the abscissa represents the interval during which the stimulus was present. The black trace shows the response when the stimulus was presented in the receptive field of the neuron in the condition where the animal did not make a saccade. The green trace shows the response when the stimulus was presented in the presaccadic receptive field of the neuron and a saccade was made; the blue trace shows the same but with the stimulus presented in the postsaccadic receptive field. The vertical dashed line indicates the mean time of saccadic onset.
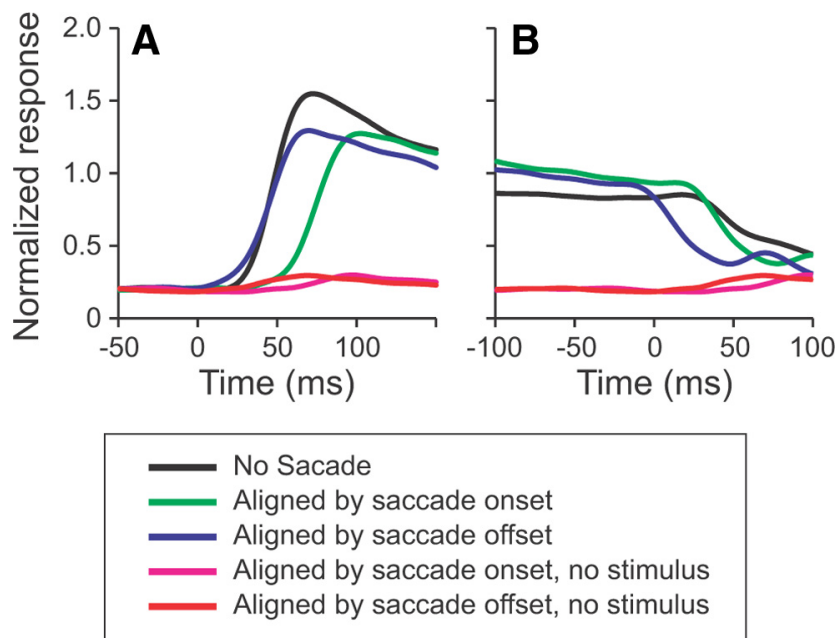

Figure 3. Mean latencies of responses as a function of stimulus onset, saccadic onset, or saccadic offset. $\boldsymbol{A}$, The mean normalized on-responses from 73 neurons. When no saccade was made, the on-response represents the initial visual response (black trace). When a saccade was made, the on-response represents the response to the stimulus brought into the receptive field by a saccade (blue and green traces). The no-stimulus traces show the responses when a saccade was made, but no stimulus was presented. $\boldsymbol{B}$, Mean normalized off-responses from the same 73 neurons under the same 5 conditions.

of an unadapted neuron by a saccade appears greater. However, this response was not as strong as the initial visual transient seen when the stimulus first appeared in RF1.

\section{Absence of perisaccadic remapping of a stable stimulus}

Although the receptive fields were clearly retinotopic, we asked whether spatiotopic processing could occur via presaccadic or anticipatory remapping (Duhamel et al., 1992). To do this, we analyzed the latencies of the neural responses under conditions in which the stimulus was presented in the receptive field and under conditions in which the stimulus was brought into the receptive field by a saccade. We found that the latency of the response to a stimulus brought into the receptive field by a saccade when aligned by the saccade offset (Fig. $3 A$, blue trace) was similar to the response latency when the stimulus was first presented in the receptive field (black trace). To quantify this, we calculated the latency of the visual on-response from individual neurons and compared it to the latency of the response aligned by saccade onset (Fig. 3A, green trace) and by saccade offset (blue trace). The

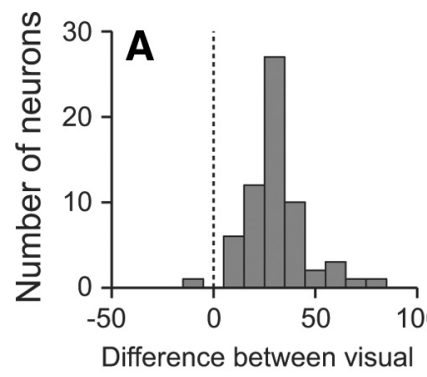

latency and response latency aligned by saccade onset (ms)

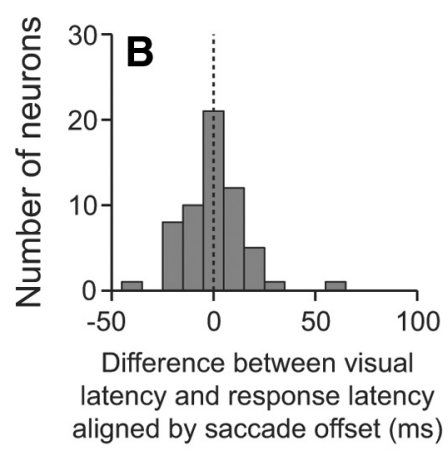

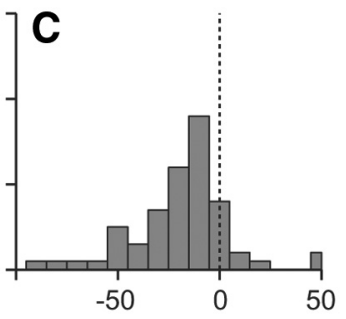

Difference between response and response end time aligned by saccade onset (ms)

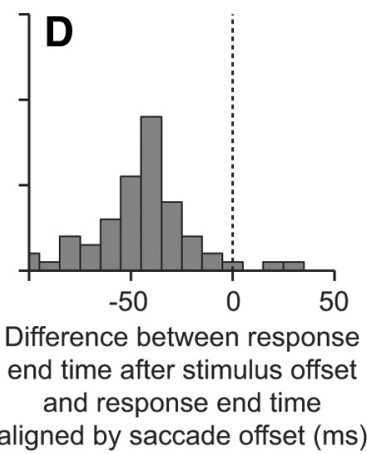
end time after stimulus offset

Figure 4. Distributions of response latencies as a function of stimulus onset and saccadic onset or saccadic offset. $A, B$, Histograms showing the difference in response latencies between the visual latency and the latency of the response aligned by saccade onset $(\boldsymbol{A})$ or offset $(\boldsymbol{B}) . \boldsymbol{C}, \boldsymbol{D}$, Histograms showing the difference in the response-end time between the end of the response caused by the stimulus being extinguished and the end of the response caused by the stimulus being removed from the receptive field by a saccade, aligned by saccade onset $(\boldsymbol{C})$ or saccade offset (D).

differences between the latencies for all neurons in which latencies could be quantified are shown in the histograms in Figure 4, $A$ and $B$. It is clear that when aligned by saccade onset (Fig. $4 A$ ), the latencies of the postsaccadic responses were significantly longer than the on-response latencies ( $p \ll 0.001, t$ test comparing mean with 0 ), with a mean difference in latency of $31.5 \pm 1.8 \mathrm{~ms}$. Only one neuron had a response latency aligned by saccade onset that was shorter than the visual latency. Thus, we can conclude that MT neurons do not show significant presaccadic remapping to a spatially stable moving stimulus. Furthermore, there was no significant difference in response latencies between the postsaccadic response aligned by saccade offset and the on-response latencies $(p=0.635)$ (Fig. $4 B$ ). From this, we interpret that there is not even a quickening of the latency, which would indicate anticipatory remapping.

To test whether the variation in alignments shown in Figure 4, $A$ and $B$, were due to the distance between receptive fields, we asked whether there was any correlation between this distance and the latency. We were unable to find any correlations between the length of the saccade or the distance between the edges of the moving dot stimulus and the alignment of neural latencies $(p>$ 0.47 ). Together, these data suggest that the responses of MT neurons are driven by light hitting the relevant photoreceptors on the retina, whether by stimulus onset or by being brought on to them by a saccade.

Another way that neural responses could produce a spatiotopic BOLD response is by maintaining information about a stimulus after a saccade has taken the stimulus out of the receptive field. To test this, we compared the drop off in activity when a stimulus was taken out of the receptive field by a saccade to the 
drop off in activity when the stimulus was turned off. The off time was defined as the time at which the spike density function first dropped below a value that was halfway between the presaccadic response and the response well after the saccade. In all cases, the neural response decreased (Fig. 3B); however, this did not occur immediately. The mean off times were $23.2 \pm 2.8 \mathrm{~ms}, 49.6 \pm 3.0$ $\mathrm{ms}$, and $66.6 \pm 3.8 \mathrm{~ms}$ when aligned by saccade offset (blue trace), saccade onset (green trace), and stimulus offset (black trace), respectively. The differences between the off times under the two saccade alignments and the off time from stimulus offset are shown in Figure 4, $C$ and $D$. There was a small but significant difference in off-response between the drop off when the stimulus was extinguished and when the saccade data were aligned by saccade onset $(p<0.001)$. Consistent with this, we found a greater difference when the saccade data were aligned by saccade offset ( $p \ll 0.001$ ). Interestingly, in both cases, the off times occurred earlier when a saccade took the stimulus out of the receptive field than when the stimulus disappeared. This is the opposite to what would be predicted if spatiotopic processing was occurring and may be consistent with a weak effect of saccadic suppression (Bremmer et al., 2009).

\section{Discussion}

Area MT has historically thought to be an area that has retinotopic receptive fields and processes information in retinotopic coordinates; however, recent studies have suggested that it may contain some spatiotopic processing (Melcher and Morrone, 2003; d'Avossa et al., 2007; Ong et al., 2009). We looked at the response of neurons in area MT to stable moving dot stimuli and found that MT neurons responded as if they had purely retinotopic receptive fields and did not show presaccadic or anticipatory remapping.

In the 40 years in which the responses of MT neurons have been recorded (Allman and Kaas, 1971), no studies have explicitly tested whether the receptive fields of MT neurons were retinotopic or spatiotopic by having animals make a saccade while a stimulus was presented in the neurons' receptive fields. This is most likely because of the robust retinotopic receptive fields seen under typical recording conditions and the fact that receptive fields appeared to be retinotopically stable when gaze was set at different locations to study gain field activity (Bremmer et al., 1997). However, given the recent suggestion that human MT has some spatiotopic selectivity (d'Avossa et al., 2007), and the psychophysical result that some processing in a memory for motion task was optimal in a spatiotopic coordinate system with MT-like receptive field sizes (Ong et al., 2009), we asked whether singleunit activity in MT had any spatiotopic processing. Consistent with our expectations, we found that receptive fields in MT were clearly retinotopic. Furthermore, we found that the timing of the activity around the time of a saccade was consistent with a visual on-response to a stable moving dot stimulus. Similar conclusions have recently been reached by a study that used a general linear model to extract receptive field information from a random noise stimulus during the slow phase of the optokinetic nystagmus and during fixation (Hartmann et al., 2011).

It is possible that the spatiotopic processing seen in the functional imaging was not due to spatiotopic receptive fields but was due to retinotopic information being passed from one neuron to another before or at the time of a saccade. Averaged out over the hemodynamic response, this may appear as a spatiotopic signal. Such remapping is found in many neurons in later cortical and subcortical areas (Duhamel et al., 1992; Walker et al., 1995; Umeno and Goldberg, 1997) and, to briefly flashed stimuli, in a number of earlier visual areas (Nakamura and Colby, 2000, 2002). We asked whether MT neurons show this remapping by comparing the onset of the visual response to the onset of the response to the stimulus as it was brought into the receptive field by a saccade. We found that almost all of the neurons showed no evidence of presaccadic remapping. Furthermore, the mean latency for the postsaccadic response aligned by saccadic offset was not different to the visual latency, suggesting that MT does not respond to a stimulus brought into its receptive field until the eye stops moving. Finally, we showed that the response to a stimulus does not linger once it has been moved out of the receptive field by a saccade. Together, we interpret these data as suggesting that there is no remapped response in MT that can explain the spatiotopic signal seen in the fMRI study.

Given that MT neurons appear to have purely retinotopic receptive fields (Hartmann et al., 2011), and no presaccadic or anticipatory remapping, one may ask: what is the genesis of the spatiotopic signal that has been seen in human area MT (d'Avossa et al., 2007)? One possible difference is the species; however, Gardner et al. (2008) have shown retinotopic processing in human MT in a task involving focused attention at the fovea. This has lead to the hypothesis that the spatiotopic signal may require peripheral attention (Burr and Morrone, 2011). In our study, the animal was neither required to carry out a task at the fovea nor the periphery, but we expect that the absence of an attention-demanding task at the fovea would appropriate sufficient attentional resources to a sudden onset stimulus in the periphery (Jonides and Irwin, 1981), giving us a response that would be similar to that in the study identifying spatiotopic processing in human MT (d'Avossa et al., 2007). This leaves the possibility that gain fields, seen in single MT neurons (Bremmer et al., 1997), may be the best explanation of the spatiotopic BOLD signal in human MT.

\section{References}

Afraz A, Cavanagh P (2009) The gender-specific face aftereffect is based in retinotopic not spatiotopic coordinates across several natural image transformations. J Vis 9:10.1-17.

Albright TD, Desimone R (1987) Local precision of visuotopic organization in the middle temporal area (MT) of the macaque. Exp Brain Res 65:582-592.

Allman JM, Kaas JH (1971) A representation of the visual field in the caudal third of the middle tempral gyrus of the owl monkey (Aotus trivirgatus). Brain Res 31:85-105.

Andersen RA, Mountcastle VB (1983) The influence of the angle of gaze upon the excitability of the light-sensitive neurons of the posterior parietal cortex. J Neurosci 3:532-548.

Bisley JW, Krishna BS, Goldberg ME (2004) A rapid and precise onresponse in posterior parietal cortex. J Neurosci 24:1833-1838.

Bremmer F (2000) Eye position effects in macaque area V4. Neuroreport 11:1277-1283.

Bremmer F, Ilg UJ, Thiele A, Distler C, Hoffmann KP (1997) Eye position effects in monkey cortex. I. Visual and pursuit-related activity in extrastriate areas MT and MST. J Neurophysiol 77:944-961.

Bremmer F, Kubischik M, Hoffmann KP, Krekelberg B (2009) Neural dynamics of saccadic suppression. J Neurosci 29:12374-12383.

Burr DC, Morrone MC (2011) Spatiotopic coding and remapping in humans. Philos Trans R Soc Lond B Biol Sci 366:504-515.

d'Avossa G, Tosetti M, Crespi S, Biagi L, Burr DC, Morrone MC (2007) Spatiotopic selectivity of BOLD responses to visual motion in human area MT. Nat Neurosci 10:249-255.

Desimone R, Ungerleider LG (1986) Multiple visual areas in the caudal superior temporal sulcus of the macaque. J Comp Neurol 248:164-189.

Duhamel JR, Colby CL, Goldberg ME (1992) The updating of the representation of visual space in parietal cortex by intended eye movements. Science 255:90-92. 
Galletti C, Battaglini PP, Fattori P (1993) Parietal neurons encoding spatial locations in craniotopic coordinates. Exp Brain Res 96:221-229.

Gardner JL, Merriam EP, Movshon JA, Heeger DJ (2008) Maps of visual space in human occipital cortex are retinotopic, not spatiotopic. J Neurosci 28:3988-3999.

Hartmann TS, Bremmer F, Krekelberg B (2011) Receptive field positions in area MT during optokinetic nystagmus.

Hays AV, Richmond BJ, Optican LM (1982) Unix-based multiple-process system, for real-time data acquisition and control. WESCON Conf Proc 2:1-10.

Jonides J, Irwin DE (1981) Capturing attention. Cognition 10:145-150.

Knapen T, Rolfs M, Cavanagh P (2009) The reference frame of the motion aftereffect is retinotopic. J Vis 9:16.

Krekelberg B, Kubischik M, Hoffmann KP, Bremmer F (2003) Neural correlates of visual localization and perisaccadic mislocalization. Neuron 37:537-545.

Maunsell JH, Gibson JR (1992) Visual response latencies in striate cortex of the macaque monkey. J Neurophysiol 68:1332-1344.

Melcher D (2005) Spatiotopic transfer of visual-form adaptation across saccadic eye movements. Curr Biol 15:1745-1748.

Melcher D, Morrone MC (2003) Spatiotopic temporal integration of visual motion across saccadic eye movements. Nat Neurosci 6:877-881.

Mirpour K, Arcizet F, Ong WS, Bisley JW (2009) Been there, seen that: a neural mechanism for performing efficient visual search. J Neurophysiol 102:3481-3491.

Morris AP, Liu CC, Cropper SJ, Forte JD, Krekelberg B, Mattingley JB (2010) Summation of visual motion across eye movements reflects a nonspatial decision mechanism. J Neurosci 30:9821-9830.
Nakamura K, Colby CL (2000) Visual, saccade-related, and cognitive activation of single neurons in monkey extrastriate area V3a. J Neurophysiol 84:677-692.

Nakamura K, Colby CL (2002) Updating of the visual representation in monkey striate and extrastriate cortex during saccades. Proc Natl Acad Sci U S A 99:4026-4031.

Ong WS, Hooshvar N, Zhang M, Bisley JW (2009) Psychophysical evidence for spatiotopic processing in area MT in a short-term memory for motion task. J Neurophysiol 102:2435-2440.

Sommer MA, Wurtz RH (2002) A pathway in primate brain for internal monitoring of movements. Science 296:1480-1482.

Umeno MM, Goldberg ME (1997) Spatial processing in the monkey frontal eye field. I. Predictive visual responses. J Neurophysiol 78:1373-1383.

Walker MF, Fitzgibbon EJ, Goldberg ME (1995) Neurons in the monkey superior colliculus predict the visual result of impending saccadic eye movements. J Neurophysiol 73:1988-2003.

Wang X, Zhang M, Cohen IS, Goldberg ME (2007) The proprioceptive representation of eye position in monkey primary somatosensory cortex. Nat Neurosci 10:640-646.

Wurtz RH (2008) Neuronal mechanisms of visual stability. Vision Res 48:2070-2089.

Zaksas D, Pasternak T (2005) Area MT neurons respond to visual motion distant from their receptive fields. J Neurophysiol 94:4156-4167.

Zhang E, Li W (2010) Perceptual learning beyond retinotopic reference frame. Proc Natl Acad Sci U S A 107:15969-15974.

Zipser D, Andersen RA (1988) A back-propagation programmed network that simulates response properties of a subset of posterior parietal neurons. Nature 331:679-684. 